\title{
Posterior versus standard approach in pancreatoduodenectomy: a case-match study
}

\author{
Traian Dumitrascu • Leonard David • Irinel Popescu
}

Received: 19 January 2009/Accepted: 17 April 2009 /Published online: 6 May 2009

(C) The Author(s) 2009. This article is published with open access at Springerlink.com

\begin{abstract}
Background and aims Posterior approach pancreatoduodenectomy (paPD) technique is one of the many modifications of the standard Whipple procedure (sPD). The most important modification of the technique is first approach of the superior mesenteric artery, thus enabling a complete dissection of the right side of this artery and of the portal vein, as well as a complete excision of the retroportal pancreatic lamina. The present study is a case-match one, comparing the paPD to sPD.

Materials and methods The present study includes two groups of patients. A first group of 21 patients with PD by posterior approach (group 1, reference group, paPD) and a second group including 21 matched patients with PD by standard approach (group 2, control group, sPD). Demographic characteristics (sex, age), intraoperative data (approach type, operative time, blood loss, intraoperative complications, need for vascular resections and type of reconstruction, type of resection upon remnant tissue), histological diagnosis and pathology data (tumor location, TNM staging, tumor grading, tumor vascular invasion), patient outcomes (postoperative length of stay, in-hospital postoperative mortality and morbidity, survival time) were analyzed in both groups of patients and compared.

Results There were no significant differences in the two groups regarding early morbidity and mortality rates, length of hospitalization, overall survival, and survival according
\end{abstract}

\footnotetext{
T. Dumitrascu $\cdot$ L. David $・$ I. Popescu $(\triangle)$

Centre of General Surgery and Liver Transplantation,

Fundeni Clinical Institute,

Fundeni Street no. 258, sector 2,

032322 Bucharest, Romania

e-mail: irinel.popescu@icfundeni.ro
}

to tumor type. However, it was noticed that there was a significant lesser mean blood loss in the pa PD group vs SPD group $(P=0.0314)$ and a shorter operative time in the paPD group vs $\mathrm{SPD}$ group $(P=0.0002)$.

Conclusion The paPD offers an early selection of patients during the operation (in terms of local resectability, by assessing the SMA infiltration), allows an optimal exposure of arterial abnormalities (a replaced right hepatic artery from the SMA), and better detection of venous invasion. In cases with PV/SMV invasion, paPD is particularly useful allowing a "no-touch" resection with no intraportal tumor dissemination and facilitates the vascular reconstruction. In addition, the operative bleeding and time are lower in the paPD group, probably due to early ligation of the inferior pancreaticoduodenal artery and reduced congestion of the pancreatic head.

Keywords Posterior approach pancreatoduodenectomy . First superior mesenteric artery approach .

"No-touch" portal vein en bloc resection · Blood loss . Operative time

\section{Introduction}

Pancreaticoduodenectomy (PD) is considered a complex operative technique, being recommended as the procedure of choice in surgical treatment of periampullary and pancreatic head tumors. PD is particularly indicated for malignant tumors (pancreas head carcinoma, malignancies of the Vater's ampulla and distal common bile duct, duodenal cancer), as the single hope for cure of these patients. Nowadays, the PD is more rarely used in the management of benign lesions; in these cases, a conservative resection is recommended when possible [1-3]. 
Since the first reports regarding the indications and technique of the $\mathrm{PD}$, this operative procedure became widely known, and many modifications and improvements of the original Whipple's procedure have been made [4-9].

Posterior approach for PD (paPD) is one of the many modifications of standard PD (sPD), and the procedure is focused on the early dissection of the posterior pancreatic capsule and first dissection of the superior mesenteric artery (SMA). The posterior part of the pancreatic head is dissected off the mesenteric vessels without dividing the pancreatic neck, thus the surgeon may avoid the "point of no return" of standard procedure and early identification of nonresectability (i.e., SMA tumor involvement). By posterior approach, a replaced right hepatic artery can be easily detected, and associated en bloc resection of the portal vein $(\mathrm{PV}) /$ superior mesenteric vein (SMV) is facilitated, using the "no-touch" technique [10-12].

There are several reports describing the operative technique of the paPD (called posterior approach, SMA first approach, etc.), but there is no paper presenting a comparative study between the paPD and SPD [10-12].

We previously described our technical version of paPD, focusing on specific and technical issues of the operation and analyzing the preliminary results for the first selected patients [12].

The aim of this paper is to present a case-match retrospective study comparing patients undergoing either paPD or sPD. The intraoperative data and postoperative outcome (including long-term survival) were compared in the two groups of patients.

\section{Materials and methods}

Patients

Between January 2003 and April 2007, 218 consecutive patients underwent PD for periampullary tumors and pancreatic head cancer at the Centre of General Surgery and Liver Transplantation, Fundeni Clinical Institute. Thus, the average number of PD per year was 51, corresponding to a high volume centre [13-15]. Of these 218 patients, a matched group of 21 patients was selected.

The present study includes two groups of patients. A first group of 21 patients with PD by posterior approach (group 1, reference group, paPD) and a second group including 21 matched patients with $\mathrm{PD}$ by standard approach (group 2, control group, sPD). Patient selection into the reference group was done due to the preference of the senior surgeon (I. Popescu) for this type of approach; patients from the control group were selected from SPD performed either by the senior surgeon (I. Popescu) or by surgeons with the same expertise in performing $\mathrm{SPD}$. The expertise in SPD is higher than in paPD. The percentage of paPD out of 218 PD was $9.63 \%$.

Patient's data were obtained by retrospective review from a prospectively gathered database at the Centre of General Surgery and Liver Transplantation of Fundeni Clinical Institute.

The matching variables were age, sex, location and type of the tumor, associated vascular resections, and follow-up time from surgery. Age and sex are usual matching variables, location of the tumor is considered to be highly important in survival studies, associated vascular resections sum up to the advanced stage of the disease, and time from surgery is selected in order to obtain similar follow-up intervals for the two groups. Assessment in cases with PV/ SMV invasion was done preoperatively by dynamic contrast-enhanced computed tomography and confirmed by intraoperative exploration. The follow-up was complete for all patients, the duration of follow-up being similar in both groups (range, 19 to 45 months). As shown in Table 1, age, sex, location and type of the tumor, number of vascular resections in each group are similar in the two groups.

Demographic characteristics (sex, age), intraoperative data (approach type, operative time, blood loss, intraoperative complications, need for vascular resections and type of reconstruction, type of resection upon remnant
Table 1 Distribution of the matching variables in the two groups

NS not significant

\begin{tabular}{llll}
\hline Factor & Group 1 (paPD) & Group 2 (sPD) & $P$ value \\
\hline Age (years) & $56( \pm 14.88)$ & $59.19( \pm 7.99)$ & $0.3918, \mathrm{NS}$ \\
Sex & \multicolumn{2}{c}{} \\
$\quad$ Male & 9 & 9 & $1, \mathrm{NS}$ \\
Female & 12 & 12 & \\
Tumour location & & & $1, \mathrm{NS}$ \\
Pancreatic head & 11 & 11 & $1, \mathrm{NS}$ \\
Ampulloma & 6 & 6 & $1, \mathrm{NS}$ \\
Distal choledocus & 3 & 4 & $1, \mathrm{NS}$ \\
Duodenum & 1 & 0 & $1, \mathrm{NS}$ \\
Vascular resection and reconstruction (PV/SMV) & 3 & 3 & \\
\hline
\end{tabular}


tissue), histological diagnosis and pathology data (tumor location, TNM staging, tumor grading, tumor vascular invasion), patients outcomes (postoperative length of stay, in-hospital postoperative mortality and morbidity, survival time) were analyzed in both groups of patients and compared.

\section{Surgical technique}

Standard technique of PD (SPD) is quite similar in most of the surgical centers and was described elsewhere [1, 3, 4, 6, 7]. Briefly, after the incision is made, the abdominal cavity is carefully explored in order to establish the presence of liver or peritoneal metastasis (these findings preclude any resection). In $\mathrm{SPD}$, the approach to determine local resectability begins by mobilizing the right colon from the right upper quadrant. After that, the Kocher's maneuver is performed, and the third and fourth part of the duodenum is reflected, the pancreas being elevated so that a hand can be passed behind the pancreas to palpate the tumor mass. Thus, this maneuver can usually determine the tumor involvement of the SMA (resulting in ending of the procedure and conversion to a palliative surgery) or $\mathrm{PV} / \mathrm{SMV}$ invasion (resulting in performing vascular resection and reconstruction). If the SMA is not involved, the lesser sac is then entered; the anterior surface of the SMV is identified. After dividing the right gastroepiploic vein, anterior branch of the inferior pancreaticoduodenal vein and middle colic vein (the last one only when strictly necessary), the anterior surface of the SMV and then PV is carefully dissected from the posterior part of the pancreatic neck. The common hepatic artery is then carefully dissected, and the gastroduodenal artery is identified, ligated, and divided. The cholecystectomy is performed, and after that, the common bile duct is ligated and divided. The $\mathrm{PV}$ can be now dissected free from the pancreas at its superior edge. Depending on the type of procedure (Whipple or pylorus preserving PD), the stomach or the duodenum is transected. After complete detachment of the PV from the posterior surface of the pancreatic neck, the transection of the pancreas is made. The pancreatic head along with distal common bile duct, duodenum ( \pm distal stomach), and first jejunal loop (after mobilization of Treitz's angle) is dissected from the right side of the PV and SMV, and the operative specimen is removed. If the tumor is adherent or invades the $\mathrm{PV} / \mathrm{SMV}$, a resection may be needed with vascular reconstruction (with or without graft interposition, depending on local situation).

The technique of paPD was described elsewhere [10-12]. Briefly, an extensive Kocher's maneuver is made (up to the level of the left border of the aorta), followed by mobilization of the third duodenum to the proximity of the duodenojejunal flexure, beneath the superior mesenteric vessels and the mesentery, by mobilizing the right colon, right colic flexure, and the right portion of the transverse mesocolon. Extensive "Kocherization" allows a good exposure of the aortocaval region; the presence of enlarged lymphadenopathy reported as positive by frozen section examination preclude any curative resection. After complete mobilization of the pancreatic head, in the angle between the left border of the inferior vena cava and the superior border of the left renal vein, the origin of SMA is identified. The whole lymphatic tissue originating at this level (representing the left portion of the retroportal process) is divided by a step-by-step procedure. Using a vascular tape around the SMA, subsequently, the artery is separated from the pancreatic tissue and $\mathrm{PV}$, and the inferior and posterior pancreaticoduodenal arteries are divided. The lymph nodes and the lymphatic tissue between the PV and SMA are dissected. Whenever a replaced right hepatic artery originating from the SMA is found, the artery should be dissected and carefully protected. After reflection of the duodenum and first jejunal loop beneath the mesenteric vessels, the first jejunal arteries can be firstly divided. This surgical step is followed by complete separation of SMA from the right retroportal process; the SMA is therefore set free until its entry into the mesentery. This step of operation is very important because during this stage, the tumor involvement to the SMA can be identified (and thus preclude any resection). Further dissection in this area is performed after dissection of the hepatoduodenal ligament. The next step is dissection of the hepatoduodenal ligament with exposure of the PV. The PV is traced down to the level of the pancreatic neck by ligating and dividing the surrounding tissues; this completely exposes the suprapancreatic PV. The dissection of the PV is continued down by inferomedial traction of the duodenum and pancreatic head. The posterolateral side of the $\mathrm{PV}$ is carefully dissected, ligating the tributaries. Since there is an avascular plane between the PV and pancreatic neck, after this dissection, the posterior surface of the pancreatic neck is exposed. In case of venous invasion, the PV or SMV might be resected in accordance with invasion extent: tumors that involve less than one third of the total circumference may be resected tangentially and those with more extensive involvement may be segmentally resected with vascular reconstruction (with primary anastomosis or graft interposition). In case of PV invasion, both sides of the vein (up and down to the tumor involvement) are "looped" for proximal and distal control. In contrast with SPD, after transection of the pancreatic neck, the pancreas is at last free from the right retroportal process.

Reconstructions after resection (anastomosis of the pancreas, bile duct, and stomach/duodenum to the ascending jejunum) were made similarly in both techniques. In the present series, the anastomosis of the pancreas to the jejunum was first performed. In cases with enlarged Wirsung and fibrous remnant pancreas, a duct-to-mucosa anastomosis was 
performed using interrupted sutures; in cases with "soft" remnant pancreatic tissue and small diameter of the Wirsung, the whole pancreatic stump was implanted into the jejunum using interrupted sutures. After that, the choledocus was implanted into the jejunum using continuous suture. Depending on the type of operation (Whipple or pylorus preserving $\mathrm{PD}$ ), the stomach or the duodenum was sutured to the jejunum by continuous suture.

\section{Statistical analysis}

Data were expressed as mean \pm standard deviation (SD), unless indicated otherwise. Minimum and maximum values were provided when necessary. Komolgornov-Smirnov test was used to assess the distribution of the data. Comparison of continuous variables was performed using unpaired Student's $t$ test and, comparison of categorical variables was performed using Fisher's exact test. Survival was assessed using Kaplan-Meier method. The survival curves were compared by a log-rank test. A $P$ value less than 0.05 was considered statistically significant.

\section{Results}

Intraoperative data

In the group of paPD, the Whipple procedure was performed in 16 patients and pylorus preserving PD in five patients; in the sPD group, Whipple procedure was performed in 14 patients and pylorus preserving $\mathrm{PD}$ in seven patients $(P=0.7337, \mathrm{NS})$.

Venous vascular involvement was recorded in three patients in both groups $(P=1, \mathrm{NS})$. In the paPD group, there were two patients with SMV involvement and one patient with PV trunk invasion; in all cases, the venous resection was followed by reconstruction using Goretex ${ }^{\circledR}$ graft interposition. In the sPD group, all the three patients had PV involvement; in two patients, the resection was followed by primary end-to-end anastomosis, and in one patient, the reconstruction was done using a Goretex ${ }^{\circledR}$ graft interposition.

The mean operative time was shorter in the paPD group vs SPD group. Thus, in the paPD group, the mean operative time was $228.57 \mathrm{~min}( \pm 75.78$; range, 120 to $450 \mathrm{~min})$, in comparison with the SPD where the mean operative time was $332.38 \mathrm{~min}$ ( \pm 85.84 ; range, 180 to $510 \mathrm{~min})(P=0.0002)$.

The mean blood loss was also lesser in the paPD group vs SPD group. Thus, in the paPD group, the mean blood loss was $292.86 \mathrm{ml}( \pm 195.12$; range, 100 to $1000 \mathrm{ml})$, in comparison with the SPD where mean blood loss was $435.71 \mathrm{ml}( \pm 219.17$; range, 200 to $1000 \mathrm{ml})(P=0.0314)$.

In both groups, there was no significant intraoperative incident.
Pathology data

There are no significant differences in the two groups regarding the tumor type and location, TNM status and staging, grade of differentiation, and tumor vascular invasion. It is also underlined the fact that in both groups, there are the same number of patients with no residual tumor type of resection (R0) and with microscopically residual tumor (R1; 12 vs nine patients, $P=1$, NS). These data are very important for the impact on the long-term survival of the patients in the two groups. Thus, having the same well-known prognostic factors influencing the longterm survival in both groups, we can establish the impact of the type of approach (paPD vs SPD) on the long-term survival of the patients.

\section{Early mortality and morbidity}

Early mortality and morbidity rates were defined as in-hospital mortality and morbidity.

There were no postoperative deaths in both groups.

Postoperative complications were encountered in seven patients with paPD (33.33\%). Pancreatic fistula was defined according to the International Study Group of Pancreatic Fistula [16]. In the paPD group, pancreatic fistula was encountered in five patients: one patient grade A fistula and grade B in four patients (all conservatively managed). Other complications were: hemoperitoneum from pancreatic stump imposing reintervention for hemostasis in one patient, followed by pancreatic fistula; one patient developed postoperative upper digestive hemorrhage conservatively treated, followed by bronchopneumonia - treated with antibiotics; one patient presented graft thrombosis and imposed reintervention for dethrombosing in the first postoperative day.

Postoperative complications were encountered in nine patients with SPD (42.85\%). Pancreatic fistula was recorded in nine patients: two patients with grade A fistula and grade B in seven patients (all conservatively managed). Other complications were registered in one patient: hemoperitoneum from pancreatic stump imposing reintervention for hemostasis, followed by pancreatic fistula.

The postoperative pancreatic fistula rate was higher in the SPD group vs paPD group $(42.85 \%$ vs $23.80 \%)$, but there was no statistically significant difference between the two groups $(P=0.3264$, NS).

Even if early morbidity rate in the SPD group is higher than in the paPD group, there was no significant difference between the two groups $(P=0.7513$, NS).

Complete data regarding early morbidity in the two groups are shown in Table 2.

The mean length of hospitalization was 19.57 days $( \pm 12.89)$ in the paPD group (range, 11 to 60 days), while in 
the SPD group, the mean length of hospitalization was 19.90 days ( \pm 12.87 ; range, 10 to 60 days). There was no significant difference regarding the postoperative length of hospitalization between the two groups ( $P=0.9336$, NS).

The mortality rate in the whole 218 PD group was $3.21 \%$. The nil mortality in the matched groups is probably a matter of hazard. The morbidity rate in the whole $218 \mathrm{PD}$ group is $42.66 \%$; the postoperative pancreatic fistula rate in the whole group was $28.44 \%$ (grade A-8\%, grade B$85.5 \%$, grade $\mathrm{C}-6.5 \%$ ). The differences regarding early postoperative morbidity, mainly pancreatic fistula, are not statistically significantly different in the matched group comparing to the whole $218 \mathrm{PD}$.

\section{Patient survival}

The mean overall survival rate in the paPD group was 19.9 months $( \pm 11.93)$ and, median survival time was 20.5 months. In the sPD group, the mean overall survival rate was 20.8 months $( \pm 14.56)$, and median survival time was 18.5 months. A detailed survival rate according to the type of tumor in both groups is shown in Table 3.

There was no statistically significant difference between the two groups regarding the overall survival rate $(P=$ $0.6560, \mathrm{NS})$ and also no difference according to the tumor type: ductal adenocarcinoma of the pancreas $(P=0.9570$, NS), adenocarcinoma of the ampulla $(P=0.3711, \mathrm{NS})$, adenocarcinoma of the common bile duct ( $P=0.9176$, NS).

Patient follow-up lasted until death or until cutoff date of October 1, 2008. At the time of the last follow-up, 14 patients were still alive in the paPD group vs 12 patients in the sPD group.

\section{Discussion}

PD is still considered a challenging operative technique even nowadays, being indicated mostly for the malignancies of the pancreatic head and periampullary region (ampulla, distal common bile duct, duodenum) $[1-4,17]$.

Table 2 Early postoperative morbidity in the two groups

\begin{tabular}{lccl}
\hline Complication & paPD & sPD & $P$ value \\
\hline Pancreatic fistula & 5 & 9 & $0.3264, \mathrm{NS}$ \\
Other complications & & & $0.6628, \mathrm{NS}$ \\
Hemoperitoneum & 1 & 1 & \\
Upper digestive hemorrhage & 1 & - & \\
Bronchopneumonia & 1 & - & \\
Graft thrombosis & 1 & - & \\
\hline
\end{tabular}

NS not significant
Since the first descriptions of the PD technique by Whipple and Kausch $[8,9]$, this operation has evolved over the years and become a routine surgery in trained surgical centers $[1,2,4,17,18]$.

Many years, the mortality rate after PD was high (up to 25-35\%), but in the present time, in experienced surgical centers, it was recorded that there was a notable decrease the mortality; nowadays, an accepted mortality rate after PD must be below $5 \%[1,2,19,20]$, although there are high-caseload hospitals that report no postoperative mortality [19]. Probably, a more realistic goal for modern interdisciplinary approaches for PD in hospitals that concentrate on pancreatic surgery is a mortality rate around $2 \%[17]$.

Despite the important decrease of mortality, morbidity after PD still remains an important issue (up to 40-60\%) [2, 20]. The morbidity is mainly correlated with hemorrhage and remnant pancreatic stump "pathology".

Many technical modification of the standard PD were made, especially related to the pancreatic anastomosis (considered Achilles' heel of morbidity and mortality). The technique of pancreatic anastomosis has been highly standardized in individual centers $[1,17,21]$, but there is still no consensus about how to perform a safe pancreatic anastomosis. Some prognostic factors for pancreatic anastomosis failure have been defined: pancreatic duct diameter, pancreatic tissue texture, surgical technique (traumatic, high blood loss vs meticulous and tissue-sparing), associated multivisceral resection (including vascular and extended radical resections) [17, 22-25].

Regarding the type of operation (Whipple versus pylorus preserving PD), it was demonstrated in 2008 by a metaanalysis considering all randomized controlled trials comparing the two procedures that there is no evidence of relevant differences in mortality, morbidity, and survival between the two operations. However, it was noted that the operating time and intraoperative blood loss were significantly reduced in patients with pylorus-preserving PD [26].

Extended radical lymphadenectomy in PD has been also demonstrated by a meta-analysis considering all randomized controlled trials comparing standard versus extended lymphadenectomy that brings no benefit regarding overall survival, and there may even be a trend toward increased morbidity [27].

The paPD is a modified technique of sPD that was initially proposed by Pessaux et al. starting from the anatomical studies regarding the retroportal lamina. This approach was proposed in order to overcome some technical difficulties and to achieve an oncologically acceptable lymph node dissection. The operative technique consists mainly of early dissection of the SMA and the posterior pancreatic capsule ("SMA first approach"). Thus, some technical difficulties such as tumor infiltration of the 
SMA, PV, or SMV, lymph node dissection on the right side of the SMA and SMV, presence of a replaced right hepatic artery can be easier managed by posterior approach $[10$ 12]. Quite similar techniques were also described [28-31].

The paPD enable the surgeon to identify the nonresectable cases during the early step of the operation, thus avoiding the "point of no return" of sPD. In sPD, when there is no clear preoperative imaging for tumor vascular invasion, intraoperative assessment of local resectability (i. e., SMA, PV, or SMV invasion) is made mainly after transection of the pancreas. SMA tumor infiltration is widely accepted as a contraindication for resection [17, 31, 32]. In sPD, SMA tumor infiltration is usually remarked toward the end of resection process (after transection of the pancreas), when there is "no point of return" and thus, leading to a "margin-positive" resection (at least R1 resection or, not very seldom, even to an $\mathrm{R} 2$ resection). From this point of view, we can clearly conclude that paPD is better than SPD in early assessment of SMA tumor infiltration, thus avoiding unnecessary resections.

Infiltration of the PV or SMV is encountered in many patients, especially with pancreatic head tumors. Regarding the assessment of tumor extension into the PV/SMV, intaoperative ultrasound or even intraportal ultrasound can be very useful [33]. En bloc PV/SMV resection is nowadays considered a safe procedure with mortality and morbidity rates quite similar to the $\mathrm{SPD}[1,17,31,32,34]$. Using the paPD, the PV/SMV can be safely removed en bloc by "no touch" resection. Depending on the location and extension of tumor venous invasion, the proximal and distal edges of the PV/SMV are looped, thus facilitating en bloc resection. The key role of SMA to PV/SMV resection in PD was also underlined by other experienced surgical centers [28-31], even if there are some differences in the operative technique, compared to paPD [10-12]. Nevertheless, dissection on the right and posterior part of the SMA guides the surgeon through the critical part of the procedure, enabling him to achieve a complete en bloc tumor resection without interfering with tumor integrity ("no touch resection") [10-12, 28-31]. Regarding the impact of PV/SMV resection on the long-term survival, it seems that only in patients with "margin-negative" resections can be seen an improvement in long-term survival $[35,36]$. Direct dissection of the tumor from the PV/SMV without using the "no touch" technique could transform a potentially curative resection into a palliative one (by increased risk for intraportal tumor dissemination).

The paPD can detect early a replaced right hepatic artery originating from the SMA; in these cases, the artery must clearly be identified and safeguarded $[10-12,35,36]$. In cases of tumor invasion, resection and reconstruction of the replaced right hepatic artery can be done using gastroduodenal artery stump [31].

The paPD, by carefully and complete dissection of the retroportal lamina should lead to an increased number of R0 resections, since it was demonstrated that the medial and posterior margins are most commonly involved [37]. However, in the present study, there was no difference between the two groups regarding the number of "margin-negative" (R0) resections in ductal adenocarcinoma of the pancreas. This could also explain the same results on long-term survival in the two groups. Recent studies highlights the importance of pathological reporting and suggests that tumor growth patterns and thorough examination, but not surgical technique, determine R1 resection rates in pancreatic cancer; the same study state that most pancreatic cancer resections are R1 resections (up to 76\%) [37].

An interesting and significant difference in the present matched study was related to operative time and intraoperative blood loss. Thus, in the paPD group, both operative time and intraoperative blood loss were significantly lower than in the SPD group. The SPD, even in experienced centers, is related to quite high blood loss (mean ranging between 700 and 1,500 ml) [1, 38]. An explanation for reduced blood loss in the paPD group could be early ligation of inferior pancreaticoduodenal artery, thus reducing the congestion into the pancreatic head (along with gastroduodenal artery and portal vein tributaries ligation). The impact of early ligation of inferior pancrea-

Table 3 Survival according to the type of tumor in the two groups

\begin{tabular}{|c|c|c|c|c|c|}
\hline \multirow[t]{2}{*}{ Type of tumor } & \multicolumn{2}{|l|}{ paPD } & \multicolumn{2}{|l|}{ sPD } & \multirow[t]{2}{*}{$P$ value } \\
\hline & Mean survival rate & Median survival & Mean survival rate & Median survival & \\
\hline $\begin{array}{l}\text { Ductal adenocarcinoma of the } \\
\text { pancreatic head }\end{array}$ & 22 months $( \pm 15.57)$ & 19 months & 22 months $( \pm 16.50)$ & 21 months & $0.9570, \mathrm{NS}$ \\
\hline Adenocarcinoma of the ampulla & 17.88 months $( \pm 11.67)$ & 18 months & 22.25 months $( \pm 10.40)$ & 21 months & $0.3711, \mathrm{NS}$ \\
\hline $\begin{array}{l}\text { Adenocarcinoma of the distal } \\
\text { common bile duct }\end{array}$ & 17.33 months $( \pm 14.74)$ & 12 months & 19.40 months $( \pm 13.93)$ & 17 months & $0.9176, \mathrm{NS}$ \\
\hline
\end{tabular}

$N S$ not significant 
ticoduodenal artery on the intraoperative bleeding was also described in other studies [39, 40]. A study of Horiguchi et al. [39] describes a modified technique of sPD in which the inferior pancreaticoduodenal artery and gastroduodenal artery are ligated before ligating the corresponding afferent veins of the pancreatic head, thus a significant lower amount of bleeding was obtained compared to SPD, but with no difference regarding the operative time. Another study of Kawai et al. [40], using the preoperative computed tomography image-assessed ligation of inferior pancreaticoduodenal artery showed significant reduced blood loss, correlated with a significant shorter operative time. Even if intraoperative bleeding was reported to be a risk factor for high morbidity after PD, in the present study, despite the significant difference between the blood loss in the two groups, the postoperative morbidity rates were not statistically significantly different.

\section{Conclusion}

There is no significant difference between the paPD group and SPD group regarding the overall survival rate and also no difference according to the tumor type. Early postoperative mortality and morbidity rates in the two groups are not significantly different. However, compared to SPD, the paPD offers an early selection of patients during the operation (in terms of local resectability, by assessing the SMA infiltration), allows an optimal exposure of arterial abnormalities (a replaced right hepatic artery from the SMA) and better detect venous invasion. In cases with $\mathrm{PV} / \mathrm{SMV}$ invasion, paPD is particularly useful allowing a "no-touch" resection with no intraportal tumor dissemination and facilitates the vascular reconstruction. In addition, the operative bleeding and time are lower in the paPD group, probably due to early ligation of the inferior pancreaticoduodenal artery and reduced congestion of the pancreatic head.

Conflict of Interests The authors declare that they have no conflict of interest.

Open Access This article is distributed under the terms of the Creative Commons Attribution Noncommercial License which permits any noncommercial use, distribution, and reproduction in any medium, provided the original author(s) and source are credited.

\section{References}

1. Cameron JL, Riall TS, Coleman JA, Belcher KA (2006) One thousand consecutive pancreaticoduodenectomies. Ann Surg 244:10-15. doi:10.1097/01.sla.0000217673.04165.ea
2. Ionescu M, Stroescu C, Barbuta S, Ciurea S, Popescu I (2003) Pancreatoduodenectomy - routine surgery? Chirurgia (Bucur) 98:103-108

3. Bachellier P, Tierris J, Wener JC, Pai M (2006) Current Practice in Pancreatic Surgery. In Habib NA and Canelo R (eds.) Liver and Pancreatic Diseases Management. Springer, pp 112-5

4. Ferrone CR, Brennan MF (2008) The Kausch-Whipple Pancreatectomy. In Beger HG, Matsuno S, and Cameron JL (eds.) Diseases of the Pancreas - Current Surgical Therapy. Springer, pp 567-79

5. Howard JM (1997) Historical Aspects and the Future of Pancreatoduodenectomy. In Hanyu $\mathrm{F}$ and Takasaki K (eds.) Pancreatoduodenectomy. Springer, pp 3-10

6. Schulick RD, Cameron JL (2008) Pylorus-Preserving Pancreatoduodenectomy. In Beger HG, Matsuno S, and Cameron JL (eds.) Diseases of the pancreas - current surgical therapy. Springer, pp $581-91$

7. Traverso LW, Longmire WP (1978) Preservation of the pylorus in pancreatoduodenectomy. Surg Gynecol Obstet 146:959-962

8. Whipple AO, Parson WB, Mullins CR (1935) Treatment of carcinoma of the ampulla of Vater. Ann Surg 102:763-779. doi:10.1097/00000658-193510000-00023

9. Kausch W (1912) Das Carcinom der Papilla Duodeni und seine radikale Entfernung: beitrage klinischen. Chirurgie 78:439-486

10. Pessaux P, Regenet N, Arnaud JP (2003) Resection of the retroportal pancreatic lamina during pancreatoduodenectomy: first dissection of the superior mesenteric artery. Ann Chir 128:633636. doi:10.1016/j.anchir.2003.10.010

11. Pessaux P, Varma D, Arnaud JP (2006) Pancreatoduodenectomy: superior mesenteric artery first approach. J Gastrointest Surg 10:607-611. doi:10.1016/j.gassur.2005.05.001

12. Popescu I, David L, Dumitra AM, Dorobantu B (2007) The posterior approach in pancreatoduodenectomy: preliminary results. Hepatogastroenterology 54:921-926

13. Ho V, Heslin MJ (2003) Effect of hospital volume and experience on in-hospital mortality for pancreaticoduodenectomy. Ann Surg 237:509-514. doi:10.1097/00000658-200304000-00012

14. Meguid RA, Ahuja N, Chang DC (2008) What constitutes a "high-volume" hospital for pancreatic resection? J Am Coll Surg 206:622-628. doi:10.1016/j.jamcollsurg.2007.11.011

15. Tjarda van Heek N, Kuhlmann KFD, Scholten RJ, de Castro SMM, Busch OR, van Gulik TM, Obertop H, Gouma DJ (2005) Hospital volume and mortality after pancreatic resection. A systematic review and an evaluation of intervention in The Netherlands. Ann Surg 242:781-790. doi:10.1097/01.sla.0000188462.00249.36

16. Bassi C, Dervenis C, Butturini G, Fingerhut A, Yeo C, Izbicki J, Neoptolemos J, Sarr M, Traverso W, Buchler M (2005) Postoperative pancreatic fistula: an international study group (ISGPF) definition. Surgery 138:8-13. doi:10.1016/j.surg. 2005.05.001

17. Buchler MW, Kleeff J, Friess H (2007) Surgical treatment of pancreatic cancer. J Am Coll Surg 205:S81-S86. doi:10.1016/j. jamcollsurg.2007.06.332

18. Hanyu F (1997) One thousand pancreatoduodenectomies at a single institution. In Hanyu F and Takasaki K (eds.) Pancreatoduodenectomy, pp 13-21

19. Cameron JL, Ha P, Yeo CJ, Lillemoe KD, Kaufman HS, Coleman JA (1993) One hundred and forty-five consecutive pancreatoduodenectomies without mortality. Ann Surg 217:430-435. doi:10.1097/00000658-199305010-00002

20. Gouma DJ, van Geenen RCI, van Gulik TM, de Haan RJ, de Wit LT, Busch OR, Obertop H (2000) Rates of complications and death after pancreatoduodenectomy: risk factors and the impact of hospital volume. Ann Surg 232:786-795. doi:10.1097/00000658200012000-00007 
21. Z'graggen K, Uhl W, Friess H, Buchler MW (2002) How to do a safe pancreatic anastomosis. J Hepatobiliary Pancreat Surg 9:733737. doi:10.1007/s005340200101

22. Bartoli FG, Arnone GB, Ravera G, Bachi V (1991) Pancreatic fistula and relative mortality in malignant disease after pancreatoduodenectomy. Review and statistical meta-analysis regarding 15 years of literature. Anticancer Res 11:1831-1848

23. Lin JW, Cameron JL, Yeo CJ, Chao YF, Chung YT (2004) Risk factors and outcomes in postpancreatoduodenectomy pancreaticocutaneous fistula. J Gastrointest Surg 8:951-959. doi:10.1016/j. gassur.2004.09.044

24. Muscari F, Suc B, Kirzin S, Hay JM, Fourtanier G, Fingerhut A, Sastre B, Chippioni J, Fagniez PL, Radovanovic A (2006) Risk factors for mortality and intra-abdominal complications after pancreatoduodenectomy: multivariate analysis in 300 patients. Surgery 139:591-598. doi:10.1016/j.surg.2005.08.012

25. van Berge Henegouwen DI, de Wit LT, van Gulik TM, Obertop H, Gouma DJ (1997) Incidence, risk factors and treatment of pancreatic leakage after pancreaticoduodenectomy: drainage versus resection of the pancreatic remnant. J Am Coll Surg 185:18 24. doi:10.1016/S1072-7515(97) 00007-0

26. Diener M, Keukaeufer C, Schwarzer G, Seiler CM, Antes G, Knaebel HP, Buchler MW (2008) Pancreatoduodenectomy (classic Whipple) versus pylorus-preserving pancreatoduodenectomy (pp Whipple) for surgical treatment of periampullary an pancreatic carcinoma. Cochrane Database of Systematic Reviews

27. Michalski CW, Kleeff J, Wente MN, Diener M, Buchler MW, Friess H (2007) Systematic review and meta-analysis of standard and extended lymphadenectomy in pancreatoduodenectomy for pancreatic cancer. Br J Surg 94:265-273. doi:10.1002/bjs.5716

28. Leach SD, Davidson BS, Ames FC, Evans DB (1996) Alternative method for exposure of the retropancreatic mesenteric vasculature during total pancreatectomy. J Surg Oncol 61:163-165. doi:10.1002/ (SICI)1096-9098(199602) 61:2<163::AID-JSO14>3.0.CO;2-A

29. Machado MCC, Penteado S, Cunha JEM, Jukemura J, Herman P, Bacchella T, Machado MAC, Montagnini AL (2001) Pancreatic head tumors with portal vein involvement: an alternative surgical approach. Hepatogastroenterology 48:1486-1487

30. Varty PP, Yamamoto H, Farges O, Belghiti J, Sauvanet A (2005) Early retropancreatic dissection during pancreatoduodenectomy. Am J Surg 189:488-491. doi:10.1016/j.amjsurg.2005.01.007
31. Weitz J, Kienle P, Schmidt J, Friess H, Buchler MW (2007) Portal vein resection for advanced pancreatic head cancer. J Am Coll Surg 204:712-716. doi:10.1016/j.jamcollsurg.2007.01.004

32. Sasson AR, Hoffman JP, Ross EA, Kagan SA, Pingpank JF, Eisenberg BL (2002) En bloc resection for locally advanced cancer of the pancreas: is it worthwhile? J Gastrointest Surg 6:147-158. doi:10.1016/S1091-255X(01) 00063-4

33. Kaneko T, Nakao A, Inoue S, Nomoto S, Hosono J, Harada A, Nonami T, Takagi H (1995) Intraportal endovascular ultrasonography as a new diagnostic procedure in pancreatic surgery. Hepatogastroenterology 42:711-716

34. Baulieux J, Adham M, Oussoultzoglou E, De la Roche E, Berthoux N, Bourdeix O, Ducerf C (1998) La pancréatectomie pour cancer avec résection des vaiseaux rétropancréatiques est-elle justifiée? Chirurgie 123:438-444. doi:10.1016/S0001-4001(99) 80069-5

35. Nakao A, Takeda S, Sakai M, Kaneko T, Inoue S, Sugimoto H, Kanazumi N (2004) Extended radical resection versus standard resection for pancreatic cancer: the rationale for extended radical resection. Pancreas 28:289-292. doi:10.1097/00006676-20040400000014

36. Nakao A, Takeda S, Inoue S, Nomoto S, Kanazumi N, Sugimoto $\mathrm{H}$, Fujii T (2006) Indications and techniques of extended resection for pancreatic cancer. World J Surg 30:976-982. doi:10.1007/ s00268-005-0438-6

37. Esposito I, Kleeff J, Bergmann F, Reiser C, Herpel E, Friess H, Schirmacher P, Buchler MW (2008) Most pancreatic cancer resections are R1 resections. Ann Surg Oncol 15:1651-1660. doi:10.1245/s10434-008-9839-8

38. Buchler MW, Friess H, Wagner M, Kulli C, Wagner V, Z'graggen $\mathrm{K}$ (2000) Pancreatic fistula after pancreatic head resection. Br J Surg 87:883-889. doi:10.1046/j.1365-2168.2000.01465.x

39. Horiguchi A, Ishihara S, Ito M, Nagata H, Shimizu T, Furusawa K, Kato R, Katada K, Miyakawa S (2007) Pancreatoduodenectomy in which dissection of the efferent arteries of the head of the pancreas is performed first. J Hepatobiliary Pancreat Surg 14:575578. doi:10.1007/s00534-006-1198-x

40. Kawai M, Tani M, Ina S, Hirono S, Nishioka R, Miyazawa M, Uchiyama K, Shimamoto T, Yamaue H (2008) CLIP method (preoperative CT image-assessed ligation of inferior pancreaticoduodenal artery) reduces intraoperative bleeding during pancreatoduodenectomy. World J Surg 32:82-87. doi:10.1007/s00268-007-9305-y 